\title{
December 2016 Pulmonary Case of the Month
}

\author{
Lewis J. Wesselius, MD
}

Department of Pulmonary Medicine

Mayo Clinic Arizona

Scottsdale, AZ

\section{History of Present IIIness}

The patient is a 29-year-old man who presented to the emergency room with right-sided pleuritic chest pain, fever, cough, and progressive dyspnea over 2 weeks.

\section{Past Medical History, Social History and Family History}

He had no prior significant medical issues and had been well until 2 weeks ago. A native of India, he has been in the US for about 5 months and works at American Express. He is a nonsmoker. Family history is noncontributory.

\section{Physical Examination}

- Vitals signs: Temperature 38.0 C, Blood Pressure 155/85 mm Hg, Heart Rate 140 beats/min, Respirations 24 breaths/min

- General: Appears to be in moderate pain and respiratory distress

- Lungs: Decreased breath sounds on the right

- Heart: regular rhythm with a tachycardia

- Abdomen: unremarkable

- Extremities: unremarkable

- Neurologic: unremarkable

\section{Radiography}

His initial chest $\mathrm{x}$-ray is shown in Figure 1.

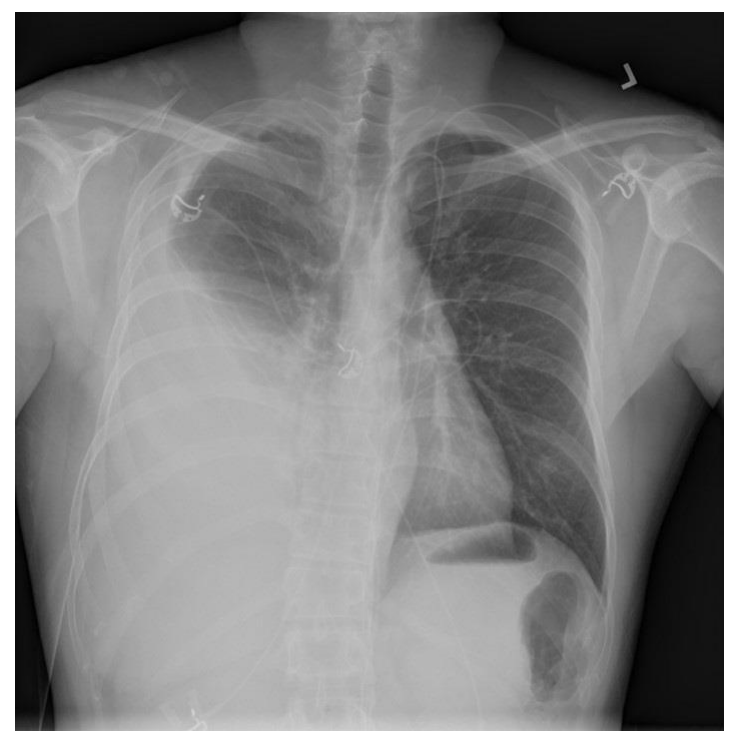

Figure 1. Initial chest radiograph. 
Which of the following best describes the chest $\mathrm{x}$-ray?

1. Elevated right hemidiaphragm

2. Large right pleural effusion

3. Right lower lobe and middle lobe consolidation

4. Right lung atelectasis

5. None of the above 


\section{Correct! \\ 2. Large right pleural effusion}

The chest $\mathrm{x}$-ray shows a large right pleural effusion. There is a concave surface at the top and laterally of the density in the right chest, a meniscus sign (Figure 2).

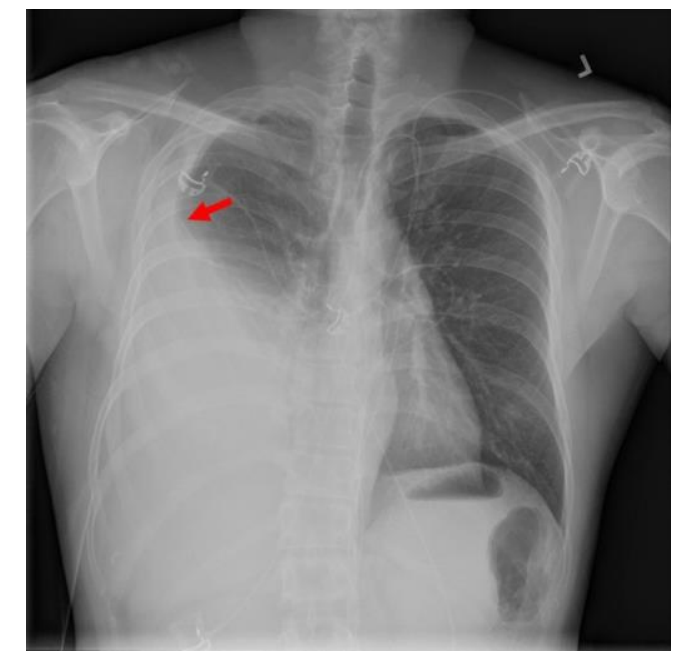

Figure 2. Meniscus sign on chest x-ray (red arrow).

The meniscus sign indicates pleural fluid extending superiorly in the pleural space and lateral to the lung. There trachea is shifted slightly to the left indicating some degree of volume expansion in the right hemithorax. Atelectasis or an elevated right hemidiaphragm would likely present with volume loss in the right chest. There are no air bronchograms (air in the bronchus surrounded by consolidated lung) which might indicate consolidation or pneumonia in the right lung.

Which of the following should be done next?

1. Coccidioidomycosis serology

2. Complete blood count (CBC) with differential

3. Liver function tests

4. Thoracic CT scan

5. All of the above 


\section{Correct! \\ 5. All of the above}

The patient is young and previously healthy and so an infectious etiology is likely. A $\mathrm{CBC}$ is indicated to look for a high white blood cell count with a left shift possibly indicative of an infection. His hemoglobin was lower than expected for a healthy, young man at $12.3 \mathrm{~g} / \mathrm{dL}$. The white blood cell count was not elevated and there was no left shift or eosinophilia. Liver function testing would be indicated since co-existing liver disease is possible but were only mildly elevated. Coccidioidomycosis or Valley Fever is very common in Arizona and a coccidioidomycosis serology is indicated but our patient's was negative. A thoracic CT would help to better define the effusion and examine the mediastinum and is shown in Figure 3.

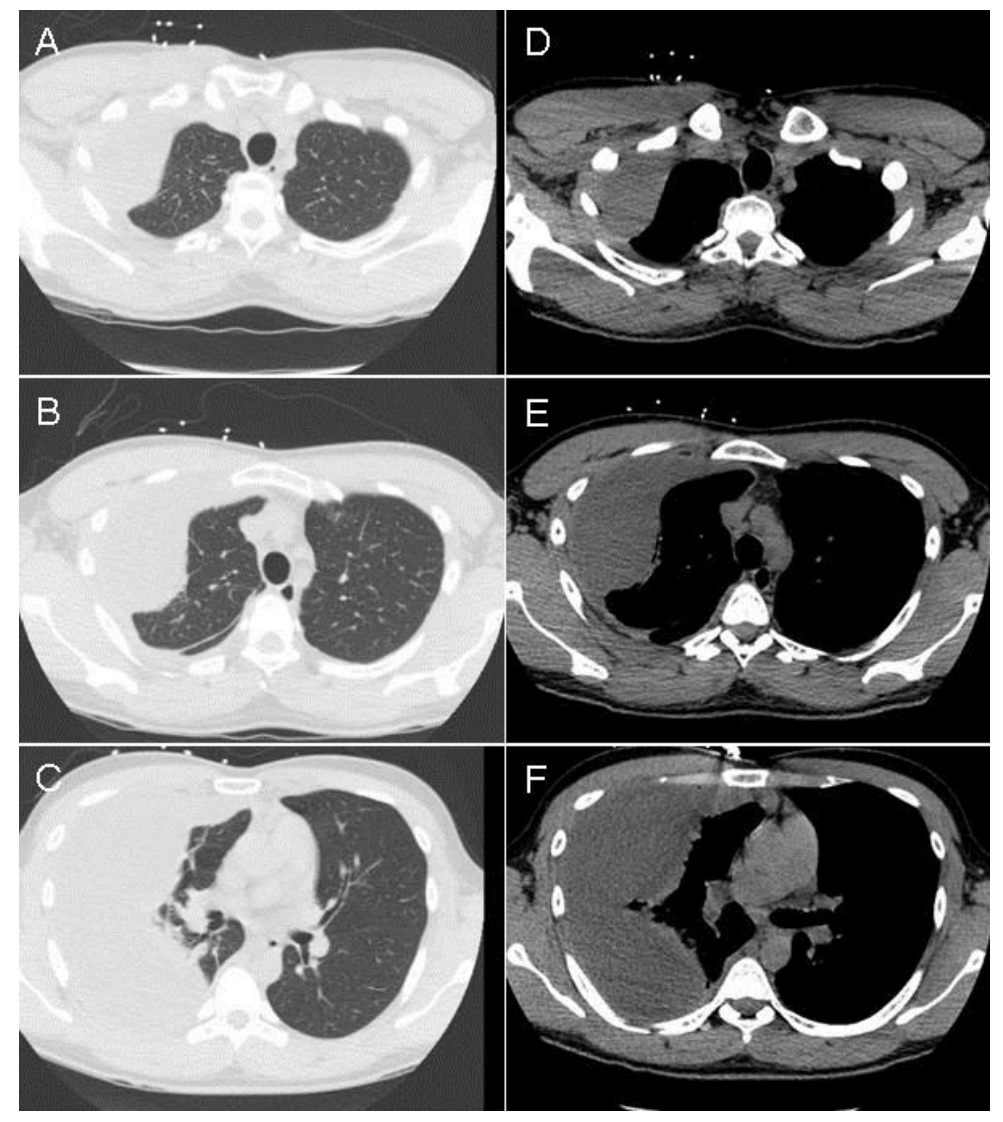

Figure 3. Representative images from thoracic CT scan in lung windows (A-C) and soft tissue windows (D-F).

Which of the following is indicated next?

1. Gold quantiferon for tuberculosis

2. Mediastinal lymph node biopsy

3. Thoracentesis

4. 1 and 3

5. All of the above 


\section{Correct!}

\section{1 and 3}

The CT scan confirms the presence of the effusion and shows little consolidation or mediastinal lymphadenopathy. Thoracentesis was performed and revealed an exudative effusion that was lymphocyte predominant on cell differential (85\%). An adenosine deaminase (ADA) was performed and slightly elevated at 9.6 U/L (normal 0-9.4). Gram stain, silver stain and acid-fast smears were negative. A PCR for tuberculosis was also negative.

The patient was a native of India where tuberculosis is common and BCG, or bacille Calmette-Guerin, a vaccine for tuberculosis is given to most. He entered the US on a work visa and unlike an immigration visa, an evaluation for tuberculosis is not required. His gold quantiferon was positive.

Which of the following is/are true?

1. A negative acid-fast smear excludes tuberculous pleuritis

2. His negative PCR for tuberculosis excludes tuberculous pleuritis

3. His positive gold quantiferon is most likely secondary to his BCG vaccination

4. Pleural fluid ADA is a sensitive test for tuberculous pleuritis.

5. None of the above 


\section{Correct! \\ 5. None of the above}

Gold quantiferon is an interferon-y (IFNy) release assay used in tuberculosis diagnosis. The patient's lymphocytes are cultured and stimulated with peptides from tuberculosis antigens. A positive result occurs when the lymphocytes release IFNy. The gold quantiferon test has higher specificity for tuberculosis than the tuberculosis skin test and is not affected by prior BCG vaccination (1).

Our patient's most likely diagnosis is tuberculosis based on his positive gold quantiferon and the clinical situation. Establishing a diagnosis of tuberculous pleuritis can be difficult (2). Tuberculous pleuritis is thought to be a delayed hypersensitivity reaction when a subpleural caseous focus ruptures into the pleural space. Therefore, the organism burden is low and cultures are positive in only 20 to $30 \%$ of cases. Furthermore, cultures can require 8-12 weeks before being considered negative.

The pleural fluid is lymphocyte predominant in 60 to $90 \%$ with the remaining being neutrophil predominant. The $\mathrm{pH}<7.3$ in $20 \%$. Our patient's pH was initially 7.33 and 7.09 on repeat. Pleural fluid glucose is usually similar to the serum level and this was the case in our patient. PCR has good specificity, but low sensitivity (46\%) and adenosine deaminase (ADA) variable sensitivity (78-99\%).

Which of the following should be done next?

1. Begin antituberculous therapy and await culture results

2. Pleural needle biopsy

3. Thorascopic pleural biopsy

4. 1 and 3 are acceptable

5. Any of the above are acceptable 


\section{Correct! \\ 5. Any of the above are acceptable}

Depending on the clinical situation, any of the above are considered acceptable. He was anxious for a diagnosis and pleuroscopy with biopsy was performed. The results show granulomas and a positive acid-fast stain (Figure 4).

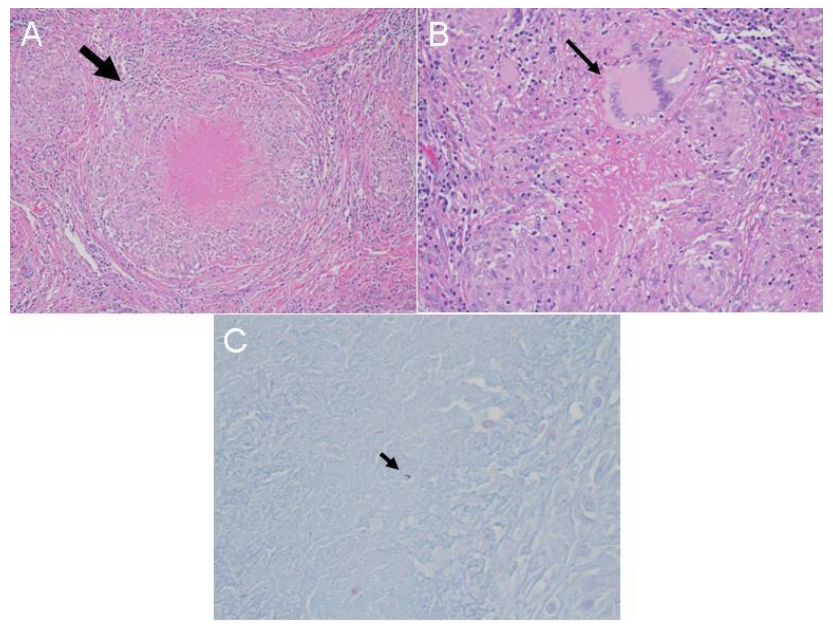

Figure 4. Histology of pleural fluid biopsy. Panel A: Low power view (H\&E) showing a granuloma with central casseous necrosis (arrow). Panel B: Higher power view (H\&E) showing a multinucleated giant cell (arrow). Panel C: High power view showing a single acid-fast organism (arrow).

Which of the following should be done next?

1. Begin 4 drug therapy while awaiting culture and sensitivity results

2. Begin corticosteroids to prevent a pleural fluid thickening with a reduction in vital capacity

3. Contact the CDC since most cases of tuberculosis in India are multi-drug resistant

4. 1 and 3

5. All of the above 


\section{Correct! \\ 1. Begin 4 drug therapy while awaiting culture and sensitivity results}

The chemotherapy for tuberculous pleuritis is the same as that for pulmonary tuberculosis. Initially 4 drugs are recommended (isoniazid $(\mathrm{INH})$, rifampin, pyrazinamide and ethambutol) until the results of drug susceptibility studies are available. Drug resistance is more frequent in India than the US but still only constitutes $1-2 \%$ of tuberculosis cases (3). Corticosteroids are controversial. Most only give corticosteroids only for severe pain (2).

Our patient was given 4 drugs and rapidly improved. His cultures were eventually positive and revealed drug-sensitive tuberculosis. He is presently receiving therapy with $\mathrm{INH}$ and rifampin and a 6 month total course with directly observed therapy is planned.

\section{References}

1. Diel R, Goletti D, Ferrara G, et al. Interferon-y release assays for the diagnosis of latent Mycobacterium tuberculosis infection: a systematic review and meta-analysis. Eur Respir J. 2011 Jan;37(1):88-99. [CrossRef] [PubMed]

2. Light RW. Update on tuberculous pleural effusion. Respirology 2010 Apr;15(3):4518. [CrossRef] [PubMed]

3. Institute of Medicine (US). Facing the Reality of Drug-Resistant Tuberculosis in India: Challenges and Potential Solutions: Summary of a Joint Workshop by the Institute of Medicine, the Indian National Science Academy, and the Indian Council of Medical Research. Washington (DC): National Academies Press (US); 2012. Available at: https://www.ncbi.nlm.nih.gov/books/NBK100386/ (accessed 11/25/16). 